\title{
Spin droplets in confined quantum Hall systems
}

\author{
E. Räsänen, ${ }^{1,2, *}$ H. Saarikoski, ${ }^{3}$ A. Harju, ${ }^{4}$ M. Ciorga, ${ }^{5}$ and A. S. Sachrajda ${ }^{6}$ \\ ${ }^{1}$ Institut für Theoretische Physik, Freie Universität Berlin, Arnimallee 14, D-14195 Berlin, Germany \\ ${ }^{2}$ European Theoretical Spectroscopy Facility (ETSF) \\ ${ }^{3}$ Kavli Institute of NanoScience, Delft University of Technology, 2628 CJ Delft, the Netherlands \\ ${ }^{4}$ Laboratory of Physics, Helsinki University of Technology, P.O. Box 4100, FI-02015 HUT, Finland \\ ${ }^{5}$ Institut für Experimentelle und Angewandte Physik, Universität Regensburg, Universitätsstraße 31, Regensburg 93040, Germany \\ ${ }^{6}$ Institute for Microstructural Sciences, National Research Council of Canada, Ottawa, Canada K1A 0R6
}

(Received 31 October 2007; published 7 January 2008)

\begin{abstract}
Two-dimensional semiconductor quantum dots are studied in the filling-factor range $2<\nu<3$. We find both theoretical and experimental evidence of a collective many-body phenomenon, where a fraction of the trapped electrons form an incompressible spin droplet on the highest occupied Landau level. The phenomenon occurs only when the number of electrons in the quantum dot is larger than $\sim 30$. We find the onset of the spin-droplet regime at $\nu=5 / 2$. This proposes a finite-geometry alternative to the Moore-Read-type Pfaffian state of the bulk two-dimensional electron gas. Hence, the spin-droplet formation may be related to the observed fragility of the $\nu=5 / 2$ quantum Hall state in narrow quantum point contacts.
\end{abstract}

DOI: 10.1103/PhysRevB.77.041302 PACS number(s): 73.21.La, 71.10.-w, 73.43.-f, 85.35.Be

The fractional quantum Hall effect at filling factor $\nu=5 / 2$ has recently attracted both experimental and theoretical interest. The $\nu=5 / 2$ state in the two-dimensional electron gas $^{1}$ (2DEG) has commonly been interpreted as a condensate of paired fermions described by a Pfaffian wave function. ${ }^{2}$ Alternative models that capture the incompressible nature of this state have been proposed using composite fermion theory. ${ }^{3}$ The non-Abelian braiding statistics involved in the excitations of the $\nu=5 / 2$ state makes it a possible candidate for topological quantum computing with high error tolerance. ${ }^{4}$ In experiments, however, the state has been found to be fragile, and it may break down in the presence of impurities or in narrow quantum point contacts. ${ }^{5}$

Finite-size counterparts of both integer and fractional quantum Hall states have been observed and characterized in confined 2D systems such as in semiconductor quantum dots (QDs). ${ }^{6}$ Due to the external confinement, these many-body states often differ considerably from the corresponding states in the $2 \mathrm{DEG}$. In a recent theoretical study, ${ }^{7}$ the QD counterpart of the $\nu=5 / 2$ state was defined, but the half-filled Landau level was shown to be poorly described by the Pfaffian.

In this work we show that in QDs the ground states in the $5 / 2 \geq \nu>2$ quantum Hall regime are characterized by fragmentation of the spin and charge densities into integer filling factor domains. This results in the formation of spin droplets (SDs), incompressible droplets of spin-polarized electrons in the highest occupied Landau level. We find that the SD formation is a collective phenomenon interacting electrons, and it occurs only when the total number of electrons in the QD is sufficiently large $(N \gtrsim 30)$. We detect signatures of the SD formation in two distinctive quantum-transport experiments in very good agreement with our theoretical results. The emergence of SDs is in contrast with the previous understanding which assumed ground-state oscillations with a low spin polarization. ${ }^{8,9}$ As a finite-geometry alternative to the Pfaffian state, the formation of SDs may explain the fragility of the $\nu=5 / 2$ state in narrow quantum point contacts in the 2DEG. The results are therefore relevant for the proposed experiments to probe the non-Abelian characteristics of the $\nu=5 / 2$ quantum Hall state, since they make use of confined geometries. $^{10}$

We analyze many-electron ground states of QDs in magnetic fields both theoretically and experimentally. In our theoretical model we use the standard effective-mass Hamiltonian

$$
H=\sum_{i=1}^{N}\left[\frac{\left(\mathbf{p}_{i}+e \mathbf{A}\right)^{2}}{2 m^{*}}+V_{\mathrm{c}}\left(r_{i}\right)+g^{*} \mu_{B} S_{z, i}\right]+\frac{e^{2}}{4 \pi \epsilon_{i<j}} \sum_{i j} \frac{1}{r_{i j}},
$$

where $V_{\mathrm{c}}(r)=m^{*} \omega_{0}^{2} r^{2} / 2$ is the external confinement, $m^{*}=0.067 m_{e}, \epsilon=12.7 \epsilon_{0}$, and $g^{*}=-0.44$ are the effective material parameters for GaAs semiconductor medium, and $\mathbf{A}$ is the vector potential of the homogeneous magnetic field $\mathbf{B}$ perpendicular to the QD plane. The many-electron problem is solved computationally using the (spin-)density-functional theory (DFT) and the variational quantum Monte Carlo method (QMC). Numerical details of the methods in QD applications are given in Refs. 11 and 12, respectively.

Figure 1 shows the total energies of different spin states of a 48-electron QD calculated with QMC. The DFT method shows qualitatively the same behavior. Here we point out that in QDs the filling factor in the $\nu \geq 1$ regime can be approximated by a formula $\nu=2 N / N_{0 \mathrm{LL}}$, where $N_{0 \mathrm{LL}}$ is the number of electrons in the lowest Landau level (0LL). ${ }^{7}$ At magnetic fields $B<1.1 \mathrm{~T}$ the degeneracy of the manyelectron ground states with different spin polarization is high. When $B$ is increased, the partially spin-polarized states become lower in energy with respect to the $S=0$ state. Finally at $B \sim 1.2 \mathrm{~T}$ the QD reaches the highest spin polarization $(S=4)$. This state can be identified as the $\nu=5 / 2$ state, which is broken into two incompressible integer filling-factor domains $\nu=2$ and $\nu=3$ inside the QD. ${ }^{7}$ The electron occupations are separated into the spin-compensated lowest OLL, forming a relatively flat background of electrons, and into 


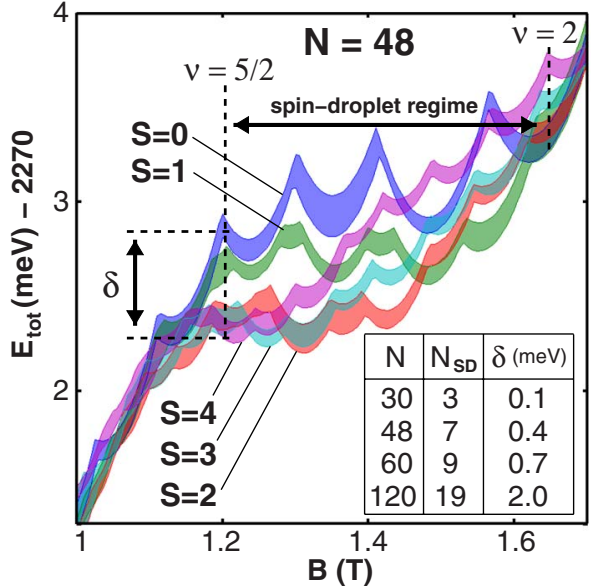

FIG. 1. (Color online) Total energies of spin states of the $N=48$ quantum dot $\left(\hbar \omega_{0}=2 \mathrm{meV}\right)$ calculated with the quantum Monte Carlo method in the spin-droplet regime. The linewidths denote the statistical error in the calculations. The table shows the density-functional-theory result for the maximum number of electrons in the spin droplet, $N_{\mathrm{SD}}$, as a function of $N$, as well as the total-energy decrease at $\nu=5 / 2$ due to the spin polarization.

the totally spin-polarized second-lowest Landau level (1LL), forming a SD. The SD gradually diminishes at $1.2<B<1.7 \mathrm{~T}$ corresponding to $5 / 2 \geq \nu>2$, which we define as the $S D$ regime. The total electron densities and the respective densities of the OLL and 1LL in the SD regime are visualized in Fig. 2. Both the DFT and QMC results show the same topology.

We define the total-energy decrease achieved from the spin polarization of the 1LL as $\delta=\max \left[E(S=0)-E\left(S_{\max }\right)\right]$ calculated at $\nu=5 / 2$-i.e., at the onset of the $\mathrm{SD}$ regime. The Zeeman energy has only a minor $(<10 \%)$ contribution in $\delta$ in these magnetic fields, the rest being due to electron-

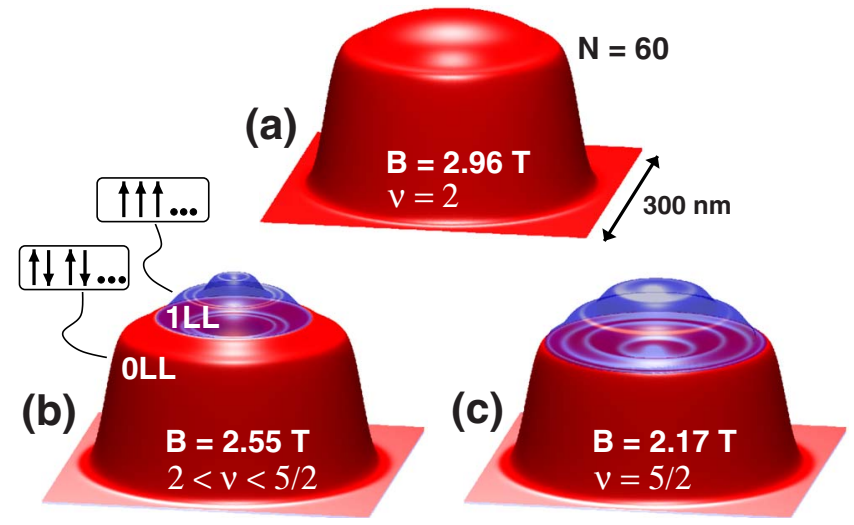

FIG. 2. (Color online) Electron densities of a 60-electron quantum dot calculated with the density-functional method at $\nu=2$ (a), at an intermediate state between $\nu=2$ and $\nu=5 / 2$ (b), and at $\nu=5 / 2$ (c). The red (solid) and blue (transparent) regions mark the electron densities in the spin-compensated lowest (OLL) and the spinpolarized second-lowest Landau levels (1LL), respectively. The series of figures thus demonstrates the formation and growth of the spin droplet in the second lowest Landau level. electron interactions. Our calculations show that SDs do not form below $N \leqslant 30$ due to the loss of spin polarization of the 1LL. The table in Fig. 1 demonstrates that the size of the SD, $N_{\mathrm{SD}}$, grows with $N$ and $\delta$ is roughly linearly proportional to $N-30$. This indicates a high stability of SDs in large QDs approaching the width of the narrow quantum point contacts made in 2DEG. ${ }^{5}$ The DFT method predicts that the SD formation is insensitive to confinement strength at least for confinements $1-4 \mathrm{meV}$.

The spin-compensated DFT calculations in the SD regime indicate a high degeneracy of single-particle (Kohn-Sham) states of the 1LL electrons near the Fermi level. The SD formation can then be understood in terms of the Stoner criterion which states that in the presence of correlations between electrons of the same spin and high density of states near the Fermi level, the system prefers ferromagnetic alignment which reduces the degeneracy. ${ }^{13}$ We note that the reduction in degeneracy from what is found in the 2DEG is due to the external confining potential.

We discuss now the evidence for the emergence of SDs in the electron transport data in two experimental setups for lateral and vertical QD devices, respectively. The emergence of finite-size counterparts of integer and fractional quantum Hall states is reflected on the energetics of the QD system. The existence and properties of these states can be probed by measuring, e.g., the chemical potential $\mu(N, B)$ $=E_{\mathrm{tot}}(N, B)-E_{\mathrm{tot}}(N-1, B)$, which is the energy required by the $N$ th electron to enter the QD. Several experimental methods have been developed for this purpose including Coulomb blockade,${ }^{14}$ capacitance, ${ }^{15}$ and charge detection techniques. ${ }^{16}$

Our setup for lateral QDs combines Coulomb and spinblockade measurements described in detail in Ref. 17. Two samples $(A$ and $B)$ of lateral devices were manufactured, and standard low-power ac measurement techniques were used: a small ac voltage with an amplitude $d V=10 \mu \mathrm{V}$ and frequency $f=23 \mathrm{~Hz}$ was applied across the sample, and a current amplifier and lock-in amplifier were used to measure the resulting spin-resolved current up to $N=48 .{ }^{17}$ The bulk mobility of the $\mathrm{Al}_{x} \mathrm{Ga}_{1-x} \mathrm{As} / \mathrm{GaAs}$ wafer used was $2 \times 10^{6} \mathrm{~cm}^{2} /(\mathrm{V} \mathrm{s})$. Another set of data was obtained from the electron transport experiments of a vertical QD device by Oosterkamp and co-workers, ${ }^{18}$ who measured the Coulombblockade oscillations up to $N=39$.

Figure 3 shows the DFT results for chemical potentials in comparison with the experiments when $N$ is between 24 and 48. The confinement strength ranged from 2 to $4 \mathrm{meV}$ depending on the electron number. The oscillations in $\mu$ correspond to ground-state transitions. Overall, we find an excellent qualitative agreement between the theory and experiments. At $\nu=5 / 2$ in high electron numbers there is a step feature followed by a plateau region superimposed on the oscillations. Our calculations show that the growing of the polarization energy $\delta$ with $N$ is crucial for the formation of these features. We identify them as signatures of the SD formation. In both the experimental data shown in Figs. 3(b) and 3(c) we note a gradual decay of the SD plateau at low electron numbers. This is in agreement with the theoretical data in Fig. 3(a). The decay is due to the fact that the spin polarization of the $1 \mathrm{LL}$ is lost when $N \leqslant 30$ (see the table in Fig. 1). 

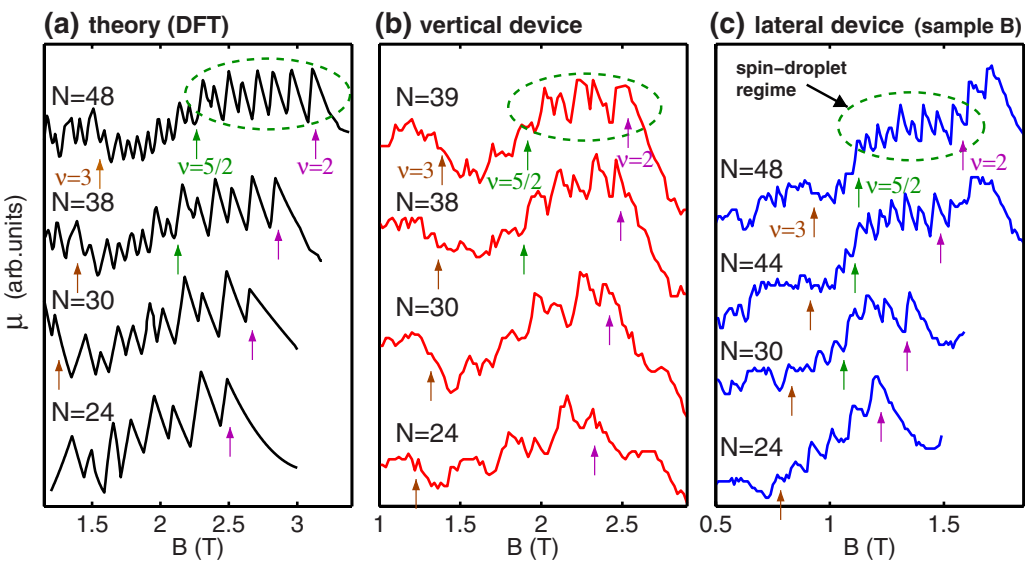

FIG. 3. (Color online) Chemical potentials calculated with density-functional theory (a) and measured from a vertical (b) and lateral (c) quantum-dot devices for various electron numbers. Both experiments show the emergence of the spin-droplet signals in the peak position data when $N \gtrsim 30$ in agreement with the theoretical result. Data for the vertical device in (b) is courtesy of L. Kouwenhoven, Delft (Ref. 18).

In Fig. 4 we compare the DFT result of the chemical potentials for $N=45$ to two samples of the lateral QDs. In the SD regime the agreement between the theory and experimental data is very good: the oscillations in $\mu$ match almost peak by peak which is an indication of discrete transitions in the many-body ground state. The corresponding values for the ground-state total angular momenta $(L)$ and spins $(S)$ ( $z$ components) calculated with the DFT are marked in Fig. 4. The similarity of the results from two different QD samples confirms our prediction of the stability of the SD even in the presence of inevitable impurities in the samples.

The electrons enter the lateral QDs from spin-polarized magnetic edge states of a 2DEG through tunneling barriers. Therefore current through the dot is enhanced if the transport involves a single-particle state in the QD which has spin parallel to the spin in the contacts. As a consequence the amplitude pattern shows a characteristic checkerboard pattern of high- and medium-conductivity regions. ${ }^{8,9}$ The conductivity pattern obtained from the DFT calculations is shown in Fig. 4 and agrees with the published amplitude

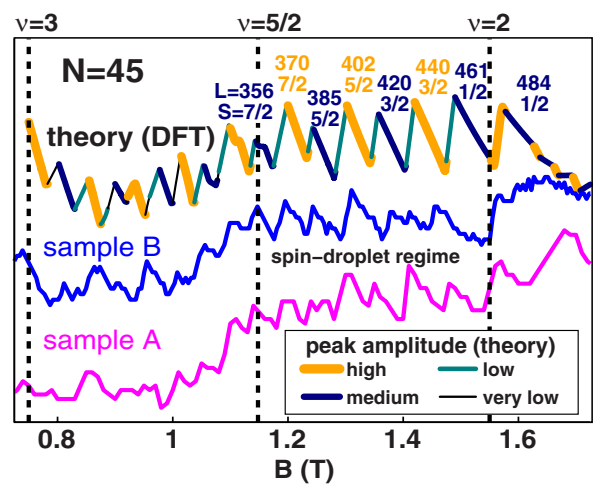

FIG. 4. (Color online) Chemical potential of two samples of lateral 45-electron dots obtained using spin-blockade spectroscopy and the corresponding theoretical result from density-functional calculations. Both samples show a clear spin-droplet plateau at 5/2 $\geq \nu>2$ in agreement with the theory. The high; medium; low; and very-low-amplitude peaks in theory correspond to transport involving the OLL spin $\uparrow$, OLL spin $\downarrow$, 1LL spin $\uparrow$, and 1LL spin $\downarrow$ states, respectively. The numbers correspond to the $z$ components of the ground-state total angular momenta $(L)$ and spins $(S)$ for the highand medium-amplitude states. data. ${ }^{17}$ The cusp in the peak position data of the lateral devices at $B=1.7 \mathrm{~T}$ is related to the chemical potential jumps in the 2 DEG. ${ }^{17}$

Our theoretical and experimental results suggest that the SD formation could be the origin of the previously observed fragility of the bulk $\nu=5 / 2$ state in, e.g., the vicinity of narrow quantum point contacts. ${ }^{5}$ In our scenario, confinement effects such as point-contact boundaries or impurities could locally form SDs with distinct phase boundaries. The formation of SD-like regions would be analogous to incompressible regions which appear in some theories of the quantum Hall effect in a disordered 2DEG. ${ }^{19}$ This type of behavior has been observed in the 2DEG by Tessmer and co-workers ${ }^{20}$ who, using scanning-probe imaging techniques, observed the formation of a system of incompressible droplets with reduced electron density in a perturbed quantum Hall liquid. In another study Finkelstein and co-workers ${ }^{21}$ used the same probing technique to map the random potential believed to be responsible for the localization of electrons near integer quantum Hall plateaus. We point out that additional theoretical studies, where the Pfaffian $\nu=5 / 2$ state is exposed to confinement, are needed to confirm this breakdown mechanism. Direct experimental evidence of the SD formation in QDs may be attained using accurate imaging methods. ${ }^{22}$

We finally point out that the large spin polarization of the SD state is encouraging for potential applications in the field of spintronics. Spin-polarized quantum Hall states such as $\nu=1$ and $\nu=1 / 3$ have been recently proposed as building blocks for spintronics devices through the spin-orbit coupling tuned by an external electric field. ${ }^{23}$ Spin-polarized electrons in SDs could be exploited in similar applications.

To conclude, we have shown evidence for spin-droplet formation in quantum dots at large electron numbers. The spin droplets are found to be largest at magnetic fields corresponding to $\nu=5 / 2$. The computational results given by two different many-electron methods agree with experimental sets of data from electron transport measurements and show that the spin-droplet formation is a collective manyelectron phenomenon. The formation of locally confined spin-droplet states in the presence of inhomogeneities such as impurities or point contacts may explain the observed fragility of the $\nu=5 / 2$ quantum Hall state in the 2DEG. Our findings stress the importance of confinement effects on quantum many-body states in attempts to realize topological quantum computing. 
We thank L. Kouwenhoven for the kind permission to reproduce their experimental data for the vertical quantumdot device and P. Zawadzki, Z. Wasilewski, and P. Hawrylak for assistance and discussions concerning the lateral dot experiments. This work was supported by the EU's Sixth
Framework Programme through the Nanoquanta Network of Excellence (NMP4-CT-2004-500198), the Academy of Finland, the Finnish Academy of Science and Letters through the Viljo, Yrjö and Kalle Väisälä Foundation, and the Canadian Institute for Advanced Research. *esa@physik.fu-berlin.de

${ }^{1}$ R. Willett, J. P. Eisenstein, H. L. Störmer, D. C. Tsui, A. C. Gossard, and J. H. English, Phys. Rev. Lett. 59, 1776 (1987).

${ }^{2}$ G. Moore and N. Read, Nucl. Phys. B 360, 362 (1991).

${ }^{3}$ C. Töke and J. K. Jain, Phys. Rev. Lett. 96, 246805 (2006).

${ }^{4}$ S. Das Sarma, M. Freedman, and C. Nayak, Phys. Rev. Lett. 94, 166802 (2005). For a recent review, see also S. Das Sarma, M. Freedman, C. Nayak, S. H. Simon, and A. Stern, arXiv:0707.1889 (unpublished).

${ }^{5}$ J. B. Miller, I. P. Radu, D. M. Zumbuhl, E. M. Levenson-Falk, M. A. Kastner, C. M. Marcus, L. N. Pfeiffer, and K. W. West, Nat. Phys. 3, 561 (2007).

${ }^{6}$ S. M. Reimann and M. Manninen, Rev. Mod. Phys. 74, 1283 (2002).

${ }^{7}$ A. Harju, H. Saarikoski, and E. Räsänen, Phys. Rev. Lett. 96, 126805 (2006).

${ }^{8}$ M. Keller, U. Wilhelm, J. Schmid, J. Weis, K. v. Klitzing, and K. Eberl, Phys. Rev. B 64, 033302 (2001).

${ }^{9}$ S. Tarucha, D. G. Austing, Y. Tokura, W. G. van der Wiel, and L. P. Kouwenhoven, Phys. Rev. Lett. 84, 2485 (2000).

${ }^{10}$ A. Stern and B. I. Halperin, Phys. Rev. Lett. 96, 016802 (2006); P. Bonderson, A. Kitaev, and K. Shtengel, ibid., 96, 016803 (2006); D. E. Feldman and A. Kitaev, ibid. 97, 186803 (2006).

${ }^{11}$ T. Torsti, T. Eirola, J. Enkovaara, T. Hakala, P. Havu, V. Havu, T. Höynälänmaa, J. Ignatius, M. Lyly, I. Makkonen, T. Rantala, K. Ruotsalainen, E. Räsänen, H. Saarikoski, and M. J. Puska, Phys. Status Solidi B 243, 1016 (2006).

${ }^{12}$ A. Harju, J. Low Temp. Phys. 140, 181 (2005).
${ }^{13}$ E. C. Stoner, Proc. R. Soc. London, Ser. A 165, 372 (1938).

${ }^{14}$ P. L. McEuen, E. B. Foxman, U. Meirav, M. A. Kastner, Y. Meir, N. S. Wingreen, and S. J. Wind, Phys. Rev. Lett. 66, 1926 (1991).

${ }^{15}$ R. C. Ashoori, H. L. Stormer, J. S. Weiner, L. N. Pfeiffer, S. J. Pearton, K. W. Baldwin, and K. W. West, Phys. Rev. Lett. 68, 3088 (1992).

${ }^{16}$ M. Field, C. G. Smith, M. Pepper, D. A. Ritchie, J. E. F. Frost, G. A. C. Jones, and D. G. Hasko, Phys. Rev. Lett. 70, 1311 (1993).

${ }^{17}$ M. Ciorga, A. S. Sachrajda, P. Hawrylak, C. Gould, P. Zawadzki, S. Jullian, Y. Feng, and Z. Wasilewski, Phys. Rev. B 61, R16315 (2000).

${ }^{18}$ The data in this filling factor and electron number range are unpublished. The description of the experimental setup as well as the data in lower electron numbers is published in T. $H$. Oosterkamp, J. W. Janssen, L. P. Kouwenhoven, D. G. Austing, T. Honda, and S. Tarucha, Phys. Rev. Lett. 82, 2931 (1999).

${ }^{19}$ D. B. Chklovskii and P. A. Lee, Phys. Rev. B 48, 18060 (1993).

${ }^{20}$ S. H. Tessmer, P. I. Glicofridis, R. C. Ashoori, L. S. Levitov, and M. R. Melloch, Nature (London) 392, 51 (1998).

${ }^{21}$ G. Finkelstein, P. I. Glicofridis, R. C. Ashoori, and M. Shayegan, Science 289, 90 (2000).

${ }^{22}$ P. Fallahi, A. C. Bleszynski, R. M. Westervelt, J. Huang, J. D. Walls, E. J. Heller, M. Hanson, and A. C. Gossard, Nano Lett. 5, 2, 223 (2005).

${ }^{23}$ See, e.g., M. Califano, T. Chakraborty, and P. Pietiläinen, Appl. Phys. Lett. 87, 112508 (2005). 